\title{
Frühe postoperative Wundinfektionen
}

\author{
M. Kalbitz ${ }^{1}$, H. von Baum ${ }^{2}$ \\ ${ }^{1}$ Klinik für Unfallchirurgie, Universitätsklinikum Ulm \\ ${ }^{2}$ Institut für Med. Mikrobiologie und Hygiene, Universitätsklinikum Ulm
}

Postoperative Wundinfektionen stellen schwerwiegende Komplikationen nach Verletzungen und traumatologischorthopädischen Eingriffen dar, sind nach Harnwegsinfekten die zweithäufigste Ursache nosokomialer Infektionen und treten infolge einer Gewebeinvasion durch pathogene Mikroorganismen auf.

Das Risiko für eine postoperative Wundinfektion wird neben der Virulenz des jeweiligen Erregers von verschiedenen patienten- und interventionsbedingten Risikofak-

\section{Einleitung}

Postoperative Wundinfektionen tragen erheblich zur Morbidität und Mortalität chirurgischer Patienten bei und stellen eine schwerwiegende Komplikation nach Verletzungen und traumatologisch-orthopädischen Eingriffen dar. Neben Virulenz und Konzentration der verursachenden Mikroorganismen sind der Immunstatus des Patienten und (implantierte) Fremdmaterialien für Art und Ausmaß der Wundinfektion entscheidend.

Die frühe postoperative Wundinfektion lässt sich einteilen in:

- Haut- und Weichteilinfektionen ohne Knochenbeteiligung

- Haut- und Weichteilinfektionen mit und ohne Implantat

- Osteitis posttraumatisch/postoperativ

- Infekt nach Fremdkörperimplantation

\section{Definition}

Die frühe postoperative Wundinfektion ist Folge einer intra- oder perioperativen oder verletzungsbedingten Gewebeinvasion durch pathogene Mikroorganismen, deren zunächst lokale Vermehrung und die daraus resultierende Gewebedestruktion. Ein weiterer Infektionsweg ist die hämatogene Streuung über einen wundfernen Infektionsort, wie etwa nach Zahnbehandlungen oder Eingriffen am Gastrointestinaltrakt. toren beeinflusst. Die „kritische Keimmasse“ und die Fähigkeit mancher Bakterien einen „Biofilm“ ausbilden zu können, spielen dabei eine sehr wichtige Rolle.

Die suffiziente Diagnostik und die Therapie einer postoperativen Wundinfektion sind für den Behandlungserfolg ausschlaggebend und unumgänglich bei der Vermeidung schwerwiegender Komplikationen wie die Ausbildung einer Sepsis, einer chronischen Osteomyelitis oder einer postinfektiösen Arthrose.

Willenegger u. Roth klassifizierten 1986 postoperative Infektionen aufgrund klinischer Erfahrungen in:

- frühe (innerhalb der ersten 2 Wochen)

- verzögerte (nach den ersten 2 Wochen)

- späte (nach den ersten 10 Wochen) Infektionen

Für die Infektion von Gelenkprothesen gelten erweiterte Zeitintervalle. Hier spricht man von einer Frühinfektion bei Infekten in den ersten 3 Monaten nach Implantation.

\section{Epidemiologie}

Postoperative Wundinfektionen stellen nach Harnwegsinfekten die zweithäufigste Ursache nosokomialer Infektionen dar. Schätzungsweise 2-5\% aller Patienten entwickeln einen postoperativen Wundinfekt abhängig von individuellen Faktoren, der Art des Eingriffs und den jeweiligen Umgebungsbedingungen der durchführenden operativen Einrichtung.

Ein wesentlicher Risikofaktor ist das Ausmaß der Kontamination des Operationsgebietes. Hier erfolgt eine Unterteilung in die Kategorien:

- 1: sauber

- 2: sauber-kontaminiert

- 3: kontaminiert

- 4: infiziert 
Tabelle 1

\begin{tabular}{|c|c|}
\hline Eingriff & $\begin{array}{l}\text { Wundinfektionsrate } \\
\text { (75\% Quantil-Risikokategorie 0-3*) }\end{array}$ \\
\hline arthroskopische Eingriffe am Kniegelenk & 0,59 \\
\hline Hüftendoprothese bei Fraktur & 4,35 \\
\hline Hüftendoprothese bei Arthrose & 1,89 \\
\hline Knieendoprothese & 1,42 \\
\hline
\end{tabular}

Abb. 1 Infektionsquellen für postoperative Wundinfektionen nach Widmer.

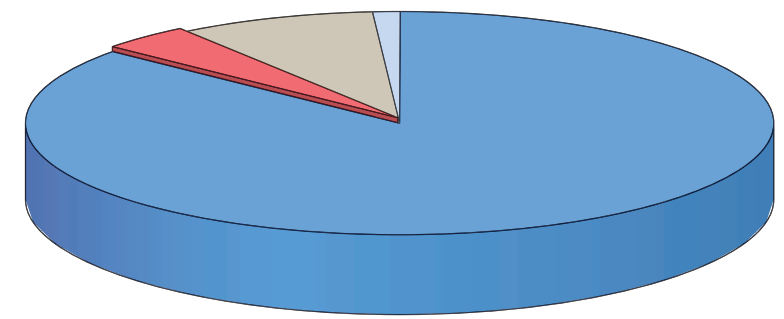

Mikroorganismen im OP-Gebiet

Mikrobielle Besiedelung des Patienten außerhalb des OP-Gebietes

Personal, Luft

Unbelebte Umgebung

Je nach Art des Eingriffes liegen die Infektionsraten für Primäreingriffe bei < $1 \%$ (nach Hüft-TEP) bis ca. $7 \%$ (kontaminiertes Gewebe); bei Revisionseingriffen steigen die Komplikationsraten erheblich an. Seit 2003 werden Wundinfektionsraten standardisiert im Modul OP-KISS des deutschen Krankenhaus-Infektions-Surveillance-Systems (KISS) erfasst (www.nrz-hygiene.de).

Die Falldefinitionen beruhen auf der CDC-Klassifikation postoperativer Wundinfektionen (Tab. 1). Die Wundinfektionsraten werden unterteilt nach Risikokategorien 0-3 (je 1 Punkt für verlängerte OP-Dauer, Wundklassifikation $>2$ und ASA-Score $>2$ ) sowie Infektionsarten A1 - A3 (oberflächlich/tief/Organinfiltration) für mehrere Indikatoroperationen jedes Fachgebietes und ermöglichen den Vergleich eigener Wundinfektionsraten mit einem nationalen Standard.

\section{Ätiologie}

Bei Infektionen wird zwischen endogenen (ca. 90\%) und exogenen (ca. 10\%) Infektionsquellen unterschieden. Exogene Infektionsquellen spielen insbesondere bei „sauberen“ Eingriffen und hier bei der Implantation von Fremdkörpern eine wichtige Rolle (Abb.1).

\section{Risikofaktoren}

Neben der Virulenz der jeweiligen Erreger beeinflusst eine Vielzahl von patienteneigenen Risikofaktoren das Auftreten einer postoperativen Wundinfektion.

\section{Zu den endogenen Risikofaktoren zählen:}

- hohes Lebensalter

- schlechter Ernährungszustand

- Diabetes mellitus

- periphere arterielle Verschlusskrankheit

- Immunsuppression

- Kortikosteroid-Einnahme

- Adipositas

- konsumierende Erkrankungen

Als exogene patientenassoziierte Risikofaktoren wurden u.a. identifiziert:

- Alkoholismus

- Nikotinabusus

- Länge des präoperativen stationären Aufenthaltes

Davon zu unterscheiden sind die interventionsassoziierten Risikofaktoren. Hierzu gehören beispielsweise:

- Länge des operativen Eingriffs

- Durchführung und der Zeitpunkt einer Rasur der Inzisionsstelle

- Operationstechnik

- perioperative Hypothermie

- Verwendung und Liegedauer von Drainagen

- Anzahl der notwendigen Revisionen

Die Rolle der perioperativ transfundierten Erythrozytenkonzentrate wird in der Literatur kontrovers diskutiert.

Das Auftreten einer postoperativen Infektion nach Frakturen wird maßgeblich vom Frakturtyp und von der Schwere des vorliegenden Weichteilschadens beeinflusst. Ein direktes Trauma kann zu Nekrosen in Weichteilen und Knochen führen und so die Kontamination mit pathogenen Organismen begünstigen. Das Risiko für eine postoperative Wundinfektion liegt bei offenen Frakturen bei 6,2\% und bei III ${ }^{\circ}$ offenen Frakturen sogar bei über $10 \%$, wohingegen bei geschlossenen Frakturen nur in 1,9\% der Fälle mit einer Infektion zu rechnen ist. 


\section{Pathogenese}

Durch eine perioperativ auftretende Gewebeschwellung und damit Erhöhung des Gewebedruckes wird die Mikrozirkulation herabgesetzt. Es kommt zu einem reduzierten Sauerstoffangebot und pH-Wert im Gewebe. Physiologische Reparaturmechanismen wie Angiogenese, Kollagenproliferation und Resorption avitalen Gewebes oder Knochens sowie die Migration von Leukozyten durch das Gefäßendothel werden gestört. Ein Circulus vitiosus beginnt.

Die „kritische Keimmasse“, d. h. die zur Auslösung einer Infektion notwendige Erregermenge, ist von individuellen Faktoren wie dem Zustand des Wundgebietes, der Immunlage des Wirtes und der Art und Virulenz des Erregers abhängig. So reichen bei kritischen Umgebungsbedingungen, wie z. B. offene Frakturen mit einliegendem Implantat, schon wenige Bakterien für den Übergang von einer Kontamination zu einer Infektion aus.

Bei Gelenkinfektionen kommt es zur Reduktion des pH-Wertes im Gelenk, Schädigung des Knorpels und später zur bakteriellen Durchwanderung in die subchondrale Region und Befall des Knochens. Parallel dazu kommt es zu einer vermehrten Flüssigkeitsproduktion durch die Synovialmembran. Der intraartikuläre Druck steigt konsekutiv an und führt weiter zu einer verminderten Durchblutung der Synovialmembran und damit zu einer weiteren Schwächung der Barriere.

Die Anwesenheit von Implantaten erhöht die lokale Infektanfälligkeit. Viele Bakterien sind zur Adhärenz auf alloplastischen Materialien fähig. Manche sind in der Lage, durch kolonieartige Zusammenlagerung und eine selbst produzierte extrazelluläre Matrix einen sog. Biofilm auszubilden (Abb. 2). Auf diese Weise können sie sich der physiologischen Infektabwehr des Wirtes und der Wirkung von Antibiotika entziehen.

Die Biofilmbildung ist von sog. "Quorum-sensing“ Molekülen abhängig, welche unter anderem die bakterielle Populationsdichte und die Anpassung an unterschiedliche Umgebungsbedingungen auf humoraler und molekulargenetischer Ebene regulieren. „Quorumsensing“ Systeme regulieren sowohl in grampositiven als auch in gramnegativen Bakterien die Expression von Adhäsionsmolekülen und Virulenzfaktoren.

Nicht zuletzt hängt das Risiko für eine postoperative Wundinfektion nach Frakturversorgung von dem gewählten Osteosyntheseverfahren ab. Bei Stabilisierung der Fraktur durch einen Fixateur externe besteht über die perkutan eingebrachten Schanz-Schrauben oder Steinmann-Nägel die Gefahr der Keimmigration. Entstehen bei dem Einbringen der Schrauben durch zu starke Hitzeentwicklung ringförmige Knochennekrosen, kann dadurch eine Keimmigration begünstigt werden.

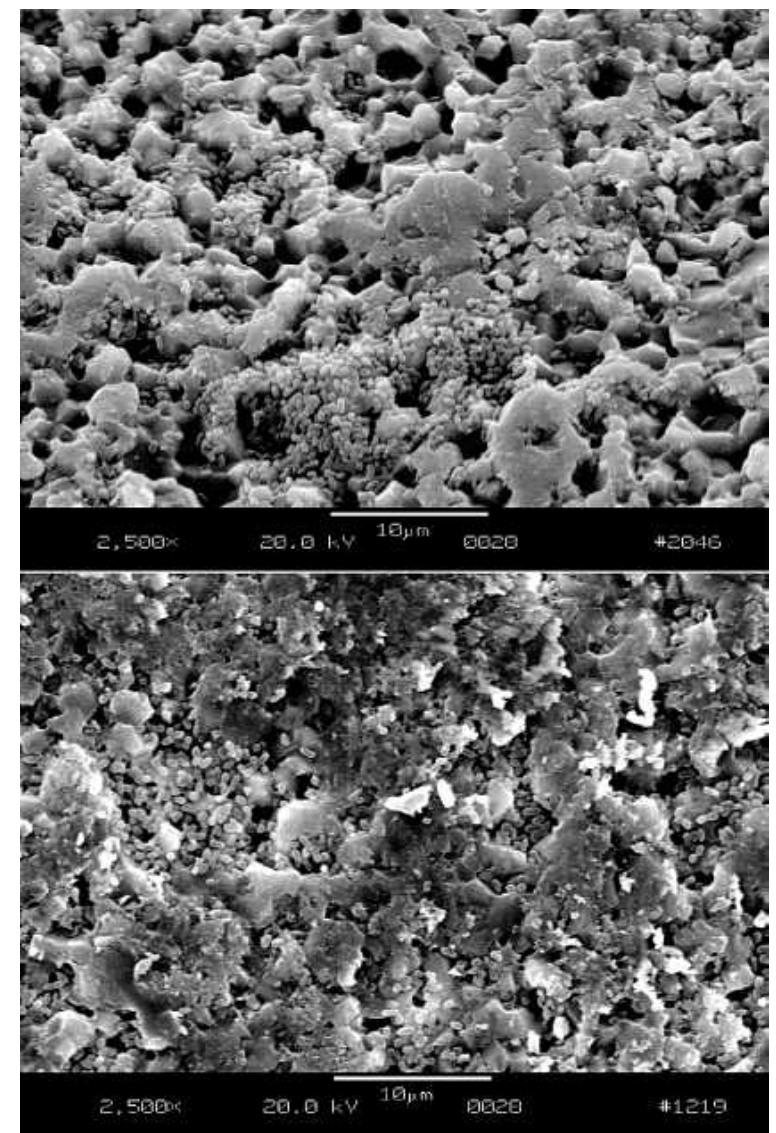

Abb. 2 - Elektronenmikroskopische Aufnahme eines Biofilms auf einer Keramik- und Edelmetalloberfläche (Abbildung mit freundlicher Genehmigung von Prof. Dr. med. Heinrich K. Geiss, Bad Neustadt).

Bei plattenosteosynthetischer Frakturversorgung kann es selbst bei korrektem operativem Vorgehen zu Devaskularisation und konsekutiv zu umschriebenen Knochennekrosen im Bereich des Plattenlagers kommen. Kommt es hierbei zu einer Infektion, kann sich diese entlang des Plattenlagers ausbreiten.

Vor allem bei der unachtsam aufgebohrten Marknagelung infolge einer zu raschen, forcierten oder exzessiven Bohrung bzw. bei Einsatz eines stumpfen Bohrers kommt es durch Hitzeentwicklung, Verschluss der Haver-Kanäle und Devaskularisation in unterschiedlichem Maße zu Knochennekrosen im Bereich der inneren Kortikalis und damit zu einer Begünstigung der Keimmigration entlang des Implantates.

Postoperativ müssen eine verzögerte Wundheilung, ischämische Nekrosen der Wundränder und ausgedehnte Hämatome von einer Infektion abgegrenzt werden, gleichzeitig sind diese Lokalbedingungen maßgeblich an der Entstehung von postoperativen Infektionen beteiligt. 


\section{Diagnostik}

Bei der Diagnostik einer frühen postoperativen Infektion stehen zunächst die klinischen Befunde und die Labordiagnostik im Vordergrund, gefolgt von bildgebenden Verfahren, die zur Diagnosesicherung herangezogen werden können.

\section{Klinik}

$\mathrm{Zu}$ den unspezifischen Allgemeinsymptomen zählen eine erhöhte Körpertemperatur, die über 3-4 Tage postoperativ hinaus anhält oder schubweise auftritt, Krankheitsgefühl, Schüttelfrost und Schweißausbrüche.

Typisch für eine lokale Infektion sind die klassischen Entzündungszeichen, die bereits vor 2000 Jahren von Celsius beschrieben wurden:

- Rubor

- Calor

- Tumor

- Dolor

- Functio laesa

Sie gelten als Kardinalzeichen der Entzündung, hervorgerufen durch mikrobielle Erreger und konsekutive Freisetzung von Entzündungsmediatoren. Daneben sind Sekretion, Druck- oder Klopfschmerzhaftigkeit oder tastbare Fluktuation Anzeichen für eine lokale Infektion. Wunddehiszenz oder Ausbildung einer Fistel können weitere Hinweise sein, speziell bei Gelenken auch das Vorhandensein eines Gelenkergusses und eine umgebende, zum Teil teigige Schwellung (Abb. 3 u. 4).

- Die sorgfältige klinische Untersuchung und Symptomanalyse hat nach wie vor die größte Bedeutung in der frühen postoperativen Infektionsdiagnostik.

\section{Labordiagnostik}

Am häufigsten werden die Parameter CRP, BSG sowie die Leukozytenzahl zur Infektdiagnostik herangezogen. Keiner dieser Parameter ist spezifisch für eine frühe postoperative Wundinfektion, da erhöhte Werte auch bei nichtinfektiösen Komplikationen auftreten können oder im Falle des CRP direkt postoperativ als Maß für das Ausmaß des intraoperativen Gewebeschadens diskutiert werden. Im direkten Vergleich ist das CRP sensitiver und spezifischer als die BSG (Tab. 2).

Beim postoperativen Monitoring werden je nach Schwere des Gewebetraumas und dem Grad der Infektion bei steigenden Infektparametern zunächst engmaschige (primär alle 24-48 Stunden) CRP-Kontrollen durchgeführt. Bei abfallenden CRP-Werten kann das Intervall entsprechend verlängert werden.

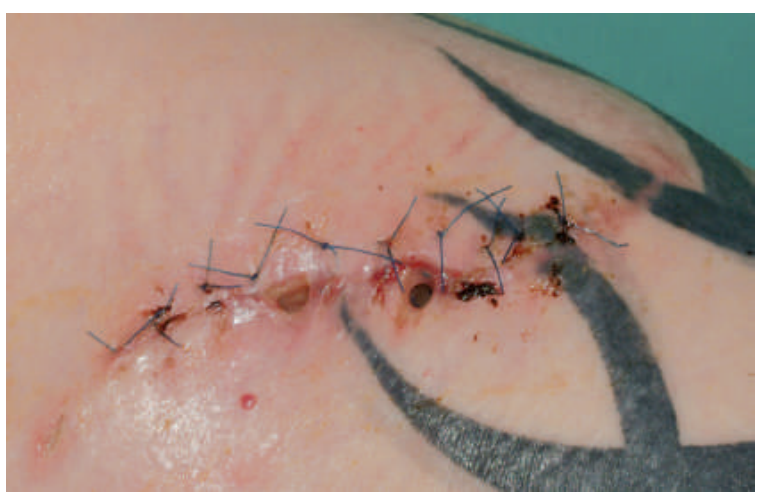

Abb. 3 - 30-jähriger Patient mit Infekt nach Tossy-III-AC-Gelenkssprengung.

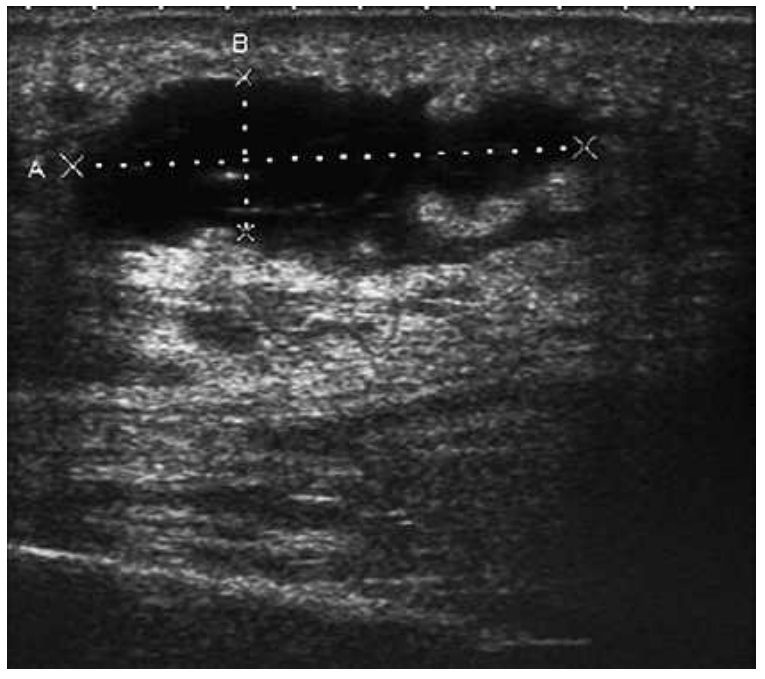

Abb. 4 - Postoperatives Serom.

\section{Invasive Diagnostik und bildgebende Verfahren}

Bei klinischem und laborchemischem Hinweis auf eine postoperative Infektion können verschiedene diagnostische und bildgebende Verfahren zur Diagnosesicherung herangezogen werden.

Sonografie. Besteht der Verdacht auf eine Gelenk- oder Weichteilinfektion, kann eine Sonografie wegweisend sein (Abb. 5). Ein Gelenkerguss, eine Verdickung der Synovialmembran, ein Hämatom oder Serom im OPGebiet sind wichtige Hinweise auf eine vorliegende Infektion. Die Sonographie ist zudem hilfreich bei der Planung diagnostischer Punktionen.

Arthroskopie. Invasiver, aber aussagekräftiger und mit dem Vorteil der gleichzeitig bestehenden Therapieoption ist bei Gelenkprozessen die Arthroskopie. Typischerweise zeigt sich eine Synovialitis purulenta bei Frühinfekt sowie Fibrinausschwitzungen und petechia- 


\begin{tabular}{|c|c|c|c|}
\hline Laborparameter & Normwert* & Charakteristik & Bewertung \\
\hline Blutkörperchensenkungsreaktion (BSR) & $\begin{array}{l}\text { Männer } \leq 15-20 \mathrm{~mm} / \mathrm{h} \\
\text { Frauen } \leq 20-30 \mathrm{~mm} / \mathrm{h}\end{array}$ & unspezifischer Suchtest & $\begin{array}{l}\text { Anstieg frühestens } 24 \mathrm{~h} \text { nach Beginn der Akute- } \\
\text { Phase-Antwort, auch bei chronisch-entzündlichen } \\
\text { Erkrankungen und Malignomen erhöht }\end{array}$ \\
\hline C-reaktives Protein (CRP) & $\leq 5 \mathrm{mg} / \mathrm{l}$ & $\begin{array}{l}\text { wichtigstes Akute-Phase-Protein } \\
\text { bei der Entzündungsdiagnostik } \\
\text { Verlaufsparameter! }\end{array}$ & $\begin{array}{l}\text { auch erhöht bei größeren Gewebedestruktionen, } \\
\text { z.B. nach operativem Eingriff, Trauma, Malignom, } \\
\text { bei Autoimmunerkrankungen oder nichtentzünd- } \\
\text { licher Akute-Phase-Reaktion, z. B. nach Lungen- } \\
\text { embolie } \\
\text { CRP-Anstieg kann der klinischen Symptomatik } \\
\text { vorausgehen }\end{array}$ \\
\hline Leukozyten & Erwachsene $3,5-10 \times 10^{9} / 1$ & $\begin{array}{l}\text { Bewertung zusammen mit } \\
\text { Differenzialblutbild }\end{array}$ & $\begin{array}{l}\text { Leukozytose mit Linksverschiebung typisch für } \\
\text { akute bakteriell-eitrige Infektionen } \\
\text { Leukopenie kann Hinweis auf gramnegative } \\
\text { Sepsis/Schock sein }\end{array}$ \\
\hline Pro-Calcitonin (PCT) & $<0,5 \mu \mathrm{g} / \mathrm{l}$ & $\begin{array}{l}\text { Serummarker insbesondere für } \\
\text { bakterielle Infektionen }\end{array}$ & $\begin{array}{l}\text { Korrelation zwischen Schweregrad der Erkrankung } \\
\text { und Serumwerten }\end{array}$ \\
\hline $\begin{array}{l}\text { Pro-inflammatorische Zytokine } \\
\text { - Interleukin } 6 \text { (IL-6) } \\
\text { - Interleukin } 8 \text { (IL-8) }\end{array}$ & $\begin{array}{l}\text { IL- } 6<10 \mathrm{pg} / \mathrm{ml} \text { Plasma } \\
\mathrm{IL}-8<10 \mathrm{pg} / \mathrm{ml} \text { Plasma }\end{array}$ & systemische Entzündungsmarker & $\begin{array}{l}\text { Sepsismarker insbesondere bei der neonatalen } \\
\text { Sepsis, IL-6 auch als Prognosemarker in der } \\
\text { Intensivmedizin eingesetzt }\end{array}$ \\
\hline Serumprotein-Elektrophorese & $\begin{array}{l}\text { Erwachsene } \\
\alpha 1 \text {-Globuline } 1,3-3,9 \mathrm{~g} / \mathrm{l} \\
\alpha 2 \text {-Globuline } 5,4-9,3 \mathrm{~g} / \mathrm{l} \\
\gamma \text {-Globuline } 5,8-15,2 \mathrm{~g} / \mathrm{l}\end{array}$ & $\begin{array}{l}\text { Erhöhung unterschiedlicher } \\
\text { Fraktionen im Zeitablauf }\end{array}$ & $\begin{array}{l}\text { Erhöhung der } \alpha \text {-Globulinfraktion etwa } 48 \text { - } 72 \text { h } \\
\text { nach Entzündungsbeginn } \\
\text { bei chronischen Entzündungen Erhöhung der } \\
\gamma \text {-Globuline }\end{array}$ \\
\hline
\end{tabular}

le Einblutungen. Bei der Arthroskopie besteht die Möglichkeit, Material für die mikrobiologische und histologische Aufarbeitung zu entnehmen und eine sofortige Untersuchung auf Bakterien (Gramfärbung) und Leuko-

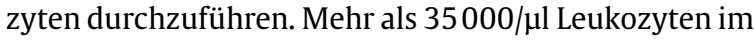
Direktpräparat gelten als Hinweis auf einen Gelenkinfekt. Das Untersuchungsmaterial kann ergänzend einer Schnellschnittdiagnostik zugeführt werden.

Röntgen. Die konventionell-radiologische Diagnostik liefert bei einer akuten postoperativen Infektion nur indirekt - z. B. durch Darstellung von Lufteinschlüssen in den Weichteilen (gasbildende Bakterien) - Anhaltspunkte für eine Infektion, gibt aber Auskunft über Frakturen, Positionierung von Implantaten oder anderen Fremdkörpern wie z. B. Beispiel Glassplitter oder Kompressen.

Kernspintomografie. Die Kernspintomografie hat sich zu einem wichtigen Bestandteil der Diagnostik postoperativer Infektionen entwickelt. Beim Vorliegen einer

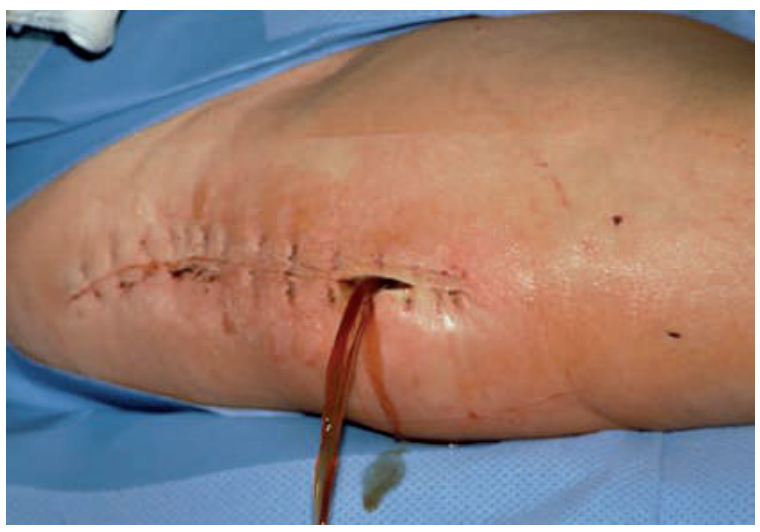

Abb. 5 - 42-jähriger Patient mit umschriebener Flüssigkeitsansammlung im suprapatellaren Weichteilgewebe nach Arthroskopie.

Infektion werden die konsekutiven Weichteilveränderungen durch veränderte Signalintensitäten, durch gesteigerten Wassergehalt (Ödem) und durch gesteigerte Kontrastmittelaufnahme abgebildet. 
Empfehlungen zur mikrobiologisch-infektiologischen Diagnostik.

\begin{tabular}{|lll}
\hline Krankheitsbild & Material & Entnahme und Transport \\
\hline hämatogene Osteomyelitis & $\begin{array}{l}\text { Blutkultur } \\
\text { (Fein-)Nadelbiopsie } \\
\text { Knochenbiopsie } \\
\text { knochen- oder prothesennahe Flüssigkeit } \\
\text { periossäre Abszessflüssigkeit }\end{array}$ & $\begin{array}{l}\text { - möglichst kontaminationsfreie Entnahme Abstriche vermeiden, da nur } \\
\text { geringe Aussagekraft } \\
\text { - Entnahme mehrerer Materialien aus einem Infektionsherd erhöht } \\
\text { diagnostische Sensitivität }\end{array}$ \\
& $\begin{array}{l}\text { - unverzüglicher Transport ins Labor (Transportzeit <2 h) } \\
\text { - Transport von Gewebeproben im sterilen Proberöhrchen bzw. in steriler } \\
\text { NaCl-Lösung }\end{array}$ \\
- Transport von Flüssigkeiten nativ z. B. in der Spritze; in Ausnahmen im \\
Blutkulturmedium
\end{tabular}

Insbesondere bei der Diagnostik von Weichteilinfekten, Myositis, Osteomyelitis, Fisteln, Fasziitis, Abszessen oder Gelenkentzündungen ist die Bildgebung mittels CT und MRT wegen ihrer hohen Auflösung und des guten Kontrastes bezüglich der Infektlokalisation und Ausdehnung allen anderen konventionellen bildgebenden Verfahren überlegen. Teilweise problematisch kann die eingeschränkte Beurteilbarkeit durch Artefakte bei einliegenden metallischen Implantaten sein.

Möglicherweise kann die Infektionsdiagnostik mittels MRT in der Zukunft durch Einsatz von USPIO (ultrasmall superparamagnetic iron oxide particles) markierten aktivierten Makrophagen noch weiter verbessert werden.

PET-CT. Durch die Möglichkeit, die exquisite Ortsauflösung des CTs (kleiner $1 \mathrm{~mm}$ ) mit der fokalen Aufnahme von 18F-FDG zu kombinieren, hat sich die PET-CT als relativ neues Verfahren bei der Diagnostik von Infektionen durchgesetzt. Der eingesetzte Tracer ist 18-Fluoro-Desoxyglucose und lässt Aussagen über den zellulären Glukosestoffwechsel zu, welcher in aktivierten Makrophagen und neutrophilen Granulozyten in entzündetem Gewebe gesteigert ist. Die Sensitivität, Spezifität und Akkuranz des PET-CTs für die Diagnostik von muskuloskelettalen Infekten liegt bei 100\%, 88\% und $93 \%$. Ein weiterer Vorteil ist die fehlende Beeinträchtigung der FDG-Aufnahme durch einliegendes Metall. Nachteilig sind die hohen Kosten, die radioaktive Belastung des Patienten und lange Wartezeiten auf Untersuchungstermine, durch die momentan noch nicht gewährleistete flächendeckende Versorgung.
Szintigrafie. Die Szintigrafie ist durch Weiterentwicklung der PET-CT-Diagnostik etwas in den Hintergrund getreten, kann aber bei der Diagnostik einer Osteomyelitis weiterhin eingesetzt werden. Die Sensitivität bei der Diagnostik einer Osteomyelitis liegt bei $73-100 \%$.

\section{Mikrobiologische/pathologische Diagnostik}

In den Empfehlungen zur mikrobiologisch-infektiologischen Diagnostik werden die folgenden Untersuchungsmaterialien und -verfahren vorgeschlagen (Tab. 3).

Von allen invasiv gewonnenen Materialien wird ein mikroskopisches Präparat angefertigt. Lassen sich hierbei Bakterien identifizieren, wird der Befund unverzüglich telefonisch übermittelt. Alle Materialien werden routinemäßig für 10 - 14 Tage bebrütet. Falls der Verdacht auf einen ungewöhnlichen Erreger (Mykobakterien, langsam wachsende Pilze) besteht, können ggf. spezifische Nährmedien bei verlängerter Bebrütungszeit eingesetzt werden. Nukleinsäure-Nachweisverfahren (PCR) sind bisher nur für einige wenige seltene Erreger von Knochen- und Gelenksinfektionen etabliert.

Bei Protheseninfektionen sollte parallel zur mikrobiologischen Aufarbeitung auch stets eine pathologische Begutachtung der Gewebeproben erfolgen. Ein interdisziplinärer und reger Informationsaustausch zwischen Klinikern, Pathologen und Mikrobiologen ist unbedingt anzustreben. 


\section{Differenzialdiagnosen}

Auch bei typischen klinischen Infektzeichen sollten differenzialdiagnostische Überlegungen stets berücksichtigt werden. So können eine aseptische Prothesenlockerung oder eine Implantatallergie (verursacht durch Nickel oder Knochenzement) ähnliche Symptome hervorrufen wie ein Infekt. Weiterhin sollten ggf. nichtinfektbedingte Ursachen wie beispielsweise Gicht, die posttraumatische oder idiopathische Arthrose oder Systemerkrankungen aus dem rheumatischen Formenkreis diskutiert werden, allerdings erst dann, wenn die Infektion sicher ausgeschlossen werden konnte.

\section{Therapie}

\section{Operative Therapie}

Der Therapieerfolg bei einer akuten postoperativen Infektion hängt ganz entscheidend vom Zeitpunkt der Intervention $\mathrm{ab}$.

Für die akute postoperative Infektion nach Osteosynthese hat sich ein Revisionskonzept durchgesetzt, bei dem die Beherrschung des Infektes dem Erhalt der Osteosynthese voranzustellen ist. Es basiert auf klinischen und mikrobiologischen Kriterien und hat 2 Ziele:

- Infektbeherrschung

- Vermeidung einer chronischen Osteomyelitis
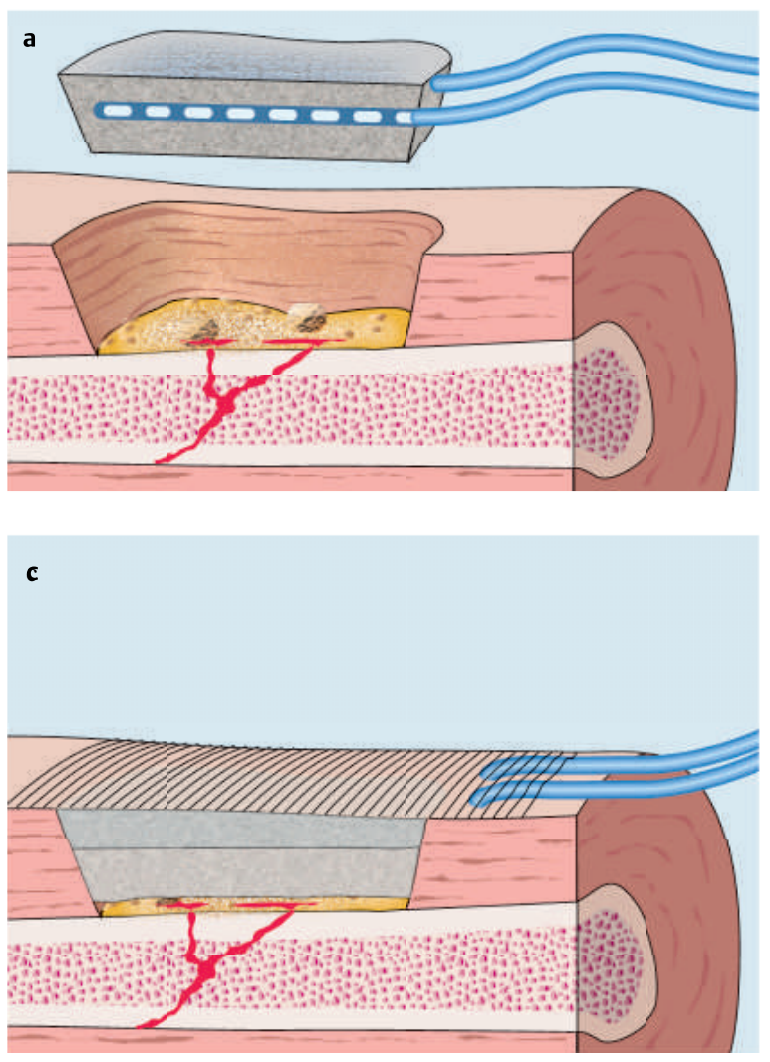

\section{Therapieziele}

\section{akute postoperative Infektion nach Osteosynthese}

- Infektbeherrschung

- Vermeidung einer chronischen Osteomyelitis

Bei Verdacht auf Frühinfekt muss die OP-Indikation dringlich gestellt werden. Bei jedem Eingriff müssen neben einem ausgiebigen Débridement eine Nekrosektomie und eine Spülung mit 0,9\%iger NaCl-Lösung erfolgen. Abstriche und Gewebeproben müssen für die mikrobiologische und histologische Aufarbeitung aus dem Implantatlager entnommen werden. Nach Erhalt der ersten Abstrichergebnisse ist die Antibiose antibiogrammgerecht anzupassen.

\section{Hintergrund}

\section{Chirurgisches Stufenschema}

- radikales Débridement, Spülung und VVS-Anlage (Abb. 6)

- second look: Nachdébridement, Spülung und VVS-Wechsel

- ggf. Entfernung des Implantats und des Fixateur externe

- Einlage von Antibiotikaträgern (antibiogrammgerecht)

- Sekundärnaht/plastische Deckung
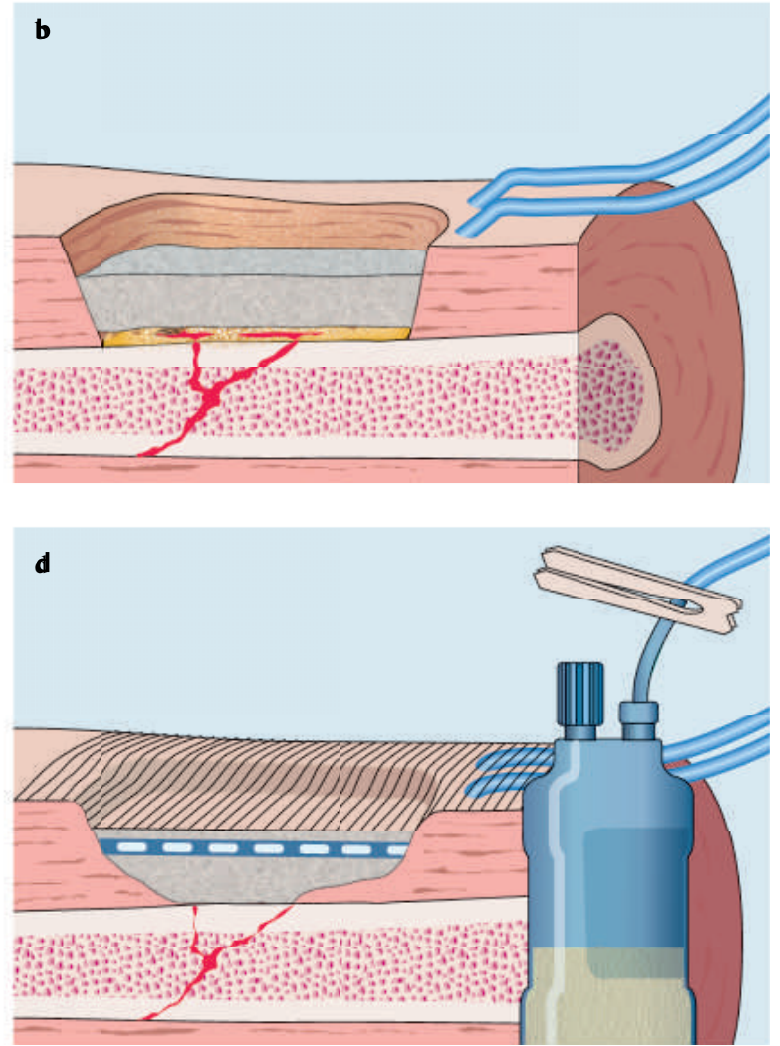

Abb. 6 =

Schematische Darstellung einer Vakuumversiegelung. a Anpassen und Einlage eines PVA-(Poly-VinylAlkohol-) Schwammes. b Ausleiten der Drainagen durch die Haut. c Steriler transparenter Folienverband (wasserdampfdurchlässig). d Anbringen einer Redon-Flasche (Unterdruck von $40-80 \mathrm{kPa}$ ). 
Spülungen mit Seifenlösung, Desinfektionsmitteln oder Antibiotikalösungen werden kontrovers diskutiert. Sie führen zwar zu einer lokalen Keimreduktion, zeigen jedoch toxische Effekte auf vitales Gewebe, werden möglicherweise unkontrollierbar resorbiert und begünstigen somit sekundär die bakterielle Besiedlung. Ebenso kritisch muss der Einsatz von Wasserdruckspülsystemen (Jet-Lavage) betrachtet werden. Zum einen wird das Gewebe durch den Wasserdruck direkt geschädigt und zum anderen besteht die Gefahr der Keimverschleppung in tiefere Gewebeschichten.

Nach o.g. chirurgischem Vorgehen können anschließend Antibiotikaträger, z.B. Kollagenvlies, eingelegt und bis zur nächsten Revision belassen werden. Der Wundverschluss wird meist unter Einlage einer Vakuumversiegelung vorgenommen. Bei konsolidierten Wundverhältnissen und fehlendem Keimnachweis in den Wundabstrichen kann ein sekundärer Wundverschluss mit Drainageneinlage erfolgen.

Können durch oben beschriebenes Vorgehen keine sterilen Wundverhältnisse erreicht werden, muss das einliegende Osteosynthesematerial entnommen werden und weiter an dem Primärziel Infektfreiheit gearbeitet werden. Die Frakturstabilisierung geschieht in diesem Fall über einen Fixateur externe. Beim Vorliegen von keimfreien Abstrichen kann eine neuerliche interne Stabilisierung erwogen werden.

Ebenso wie die Revision bei postoperativen Infekten nach Osteosynthese muss gerade die Revision bei Gelenk- oder Endoprotheseninfekten als dringlicher chirurgischer Eingriff angesehen werden.

Das Ziel ist die Infektsanierung und damit die Schmerzfreiheit und Wiederherstellung der Gelenkfunktion.

Therapieziele

\section{Gelenkinfektionen}

- Infektsanierung

- Schmerzfreiheit

- Wiederherstellung der Gelenkfunktion

Bei Gelenkinfektionen (z. B. Kniegelenk) hat zunächst die u.U. wiederholte arthroskopische Gelenkspülung zu erfolgen. Als Revisionsintervall sollen initial 24-48 Stunden nicht überschritten werden. Zwischen den Revisionen kann ein Spül-Saug-System angelegt werden. Die Ruhigstellung des Gelenkes mittels Schiene ist obligat. Im Vordergrund steht das Erzielen von 3 konsekutiven Abstrichen ohne Keimnachweis. Falls weiter Mikroorganismen im Gelenk nachgewiesen werden, muss spätestens nach 3 arthroskopischen Revisionen eine offene Gelenkrevision mit Synovektomie und Einlage von Antibiotikaträgern erfolgen. Führt dieses Vorgehen ebenfalls nicht zur Infektfreiheit, müssen der Gelenkknorpel und die Gelenkbinnenstrukturen reseziert werden. Das Gelenk wird dann über einen Fixateur externe ruhig gestellt. Eine antibiogrammgerechte Langzeitantibiose muss begonnen und für mindestens 3 Monate fortgesetzt werden

Wird ein TEP-Aufbau nach Infektsanierung geplant, sind nach Ablauf von 3 Monaten und bei befriedigendem klinischen Verlauf die Antibiose abzusetzen, Abstriche aus dem Gelenk zu entnehmen und eine Kontrolle der laborchemischen Entzündungsparameter durchzuführen. Nach Endoprothesenimplantation im Gefolge eines Infektes muss für weitere 3 Monate eine testgerechte Langzeitantibiose durchgeführt werden.

Liegt ein Endoprothesenfrühinfekt vor, ist die primäre Revision ein offenes Débridement mit Spülung, Einlage von Medikamententrägern und ggf. VAC-Anlage. Einliegende Polyethylen- und Keramikinlays müssen bei anzunehmender Keimbesiedlung entfernt und bei Keimfreiheit erneuert werden. Gleiches gilt für die Köpfe von Endoprothesen, da von einer Keimbesiedlung zwischen Kopf und Schaft ausgegangen werden muss. Einliegende metallische Komponenten werden mechanisch und chemisch gereinigt. Als Revisionsintervall sind primär ebenfalls 24-48 Stunden zu wählen. Intraoperativ besteht die Möglichkeit, Antibiotikaträger - z. B. in Form von Spacern oder Ketten - einzulegen, die mit einem Antibiotikum beschichtet wurden, das für die verursachenden Pathogene als sensibel getestet wurde. Oberstes Ziel sollte zunächst der Erhalt der Funktionsfähigkeit des Gelenkes sein.

Ist durch das beschriebene Vorgehen keine Infektfreiheit zu erzielen, muss der TEP-Ausbau erwogen werden. Dabei werden alle Prothesenanteile, nekrotischer Knochen und Zementreste entfernt und ein Spacer eingelegt. Die Spacereinlage bietet mehrere Vorteile: Die Beinlänge wird erhalten und Weichteile, Gefäße und Nerven behalten ihre ursprüngliche Anatomie.

Die Beimengung von Antibiotika in den Spacerzement kann die systemische Antibiose unterstützen. Die Gelenkfunktion kann für die physiotherapeutische Beübung erhalten werden, und verbessert somit die Funktion einer eventuellen zukünftigen Revisionsprothese.

Therapieziele

\section{Endoprothesenfrühinfekt}

Erhalt der Funktionsfähigkeit des Gelenkes 
Wichtige Voraussetzungen bei der Planung einer Revisionsprothese sind:

- Infektsanierung

- mögliche Weichteildeckung

- Compliance des Patienten

- ein ausreichendes Knochenfundament

Alternativ zur eventuellen Revisionsprothese kommt die Arthrodese in Betracht. Der Vorteil einer Arthrodese ist, je nach Verfahren, die sofortige Belastbarkeit der Extremität. Nachteilig sind die Funktionseinschränkung und die Beinlängenverkürzung.

Das Belassen einer Girdlestone-Hüfte bedeutet eine Beinverkürzung zwischen 3 und $8 \mathrm{~cm}$, je nach infektionsbedingtem Knochenverlust und eine Funktionsbeeinträchtigung oft erheblichen Ausmaßes.

Die Anlage eines Fixateur externe zur Ruhigstellung nach dem Prothesenausbau ist anspruchsvoll. Der Compliance des Patienten kommt in diesem Zusammenhang eine große Bedeutung zu.

Da die Chancen einer Erradikation bei multiresistenten Erregern äußerst gering einzuschätzen sind, muss bei Nachweis von MRSA oder MRSE der Prothesenausbau so früh wie möglich und nicht erst nach mehreren Revisionen durchgeführt werden.

\section{Antiinfektiva}

Die empirische kalkulierte Antibiotikatherapie muss unter Berücksichtigung der lokalen Resistenzsituation die häufigsten Erreger postoperativer Wundinfektionen erfassen. Dies kann im Einzelfall bedeuten, dass bereits bei der Initialtherapie problematische Erreger wie der methicillinresistene Staphylococcus aureus (MRSA) oder hochresistente Pseudomonaden bei der Wahl des Antibiotikums berücksichtigt werden müssen.

Eine Übersicht über häufige Erreger ist in Tab. 4 zusammengestellt. Es müssen Antibiotika gewählt werden, die gut in Knochen und Weichteile penetrieren und eine gute Bioverfügbarkeit aufweisen. In jedem Falle ist eine mikrobiologische Diagnostik vor Beginn der antibiotischen Therapie anzustreben. Gelingt ein Erregernachweis, so ist die antibiotische Therapie gemäß Resistogramm zu adaptieren. Empfehlungen zur Auswahl des Therapeutikums sowie zur Therapiedauer finden sich in Tab. 5.

\section{Tabelle 4}

Erreger postoperativer Gelenkinfektionen (kein Erregernachweis in $11 \%$ der Fälle).

\begin{tabular}{|l|l|}
\hline Pathogen & Häufigkeit \\
\hline koagulasenegative Staphylokokken & $30-43 \%$ \\
\hline Staphylococcus aureus & $12-23 \%$ \\
\hline Streptokokken & $9-10 \%$ \\
\hline Enterokokken & $3-7 \%$ \\
\hline gramnegative Bakterien & $3-6 \%$ \\
\hline Anaerobier & $2-4 \%$ \\
\hline Mischinfektionen & $10-11 \%$ \\
\hline
\end{tabular}

\section{Komplikationen}

Die schwerwiegendste systemische Komplikation einer postoperativen Infektion ist die Ausbildung einer Sepsis.

Die schwerwiegendste lokale Komplikation ist der Übergang in eine chronische Osteomyelitis, mit unter Umständen rezidivierender Fistelbildung und häufig schwer zu beherrschenden chronischen Schmerzen.

Im Falle einer Gelenkinfektion besteht die Gefahr einer postinfektiösen Arthrose, einem irreversiblen Funktionsverlust des entsprechenden Gelenkes bis hin zur knöchernen Ankylosierung.

Durch die oft über einen längeren Zeitraum verabreichten Antibiotika kann es zu medikamentös bedingten Komplikationen wie gastrointestinalen Beschwerden, Ausbildung einer pseudomembranösen Kolitis, Anstieg der Leberwerte oder Blutbildveränderungen kommen.

\section{Prävention}

Die Empfehlungen des Robert Koch-Institutes zur Prävention postoperativer Wundinfektionen sollten in das hauseigene Procedere eingehen. Durch den routinemäßigen Einsatz einer perioperativen Antibiotikaprophylaxe und das Operieren in Operationssälen, die mit raumlufttechnischen Anlagen ausgestattet sind, konnte das intraoperative Infektionsrisiko für Eingriffe mit Gelenksersatz nachweislich reduziert werden. 


\section{Prophylaxe und Management von Komplikationen}

Tabelle 5

\begin{tabular}{|c|c|c|c|c|}
\hline Indikation & Erreger & Initialtherapie & Therapiedauer & Quelle \\
\hline postoperative Weichteilinfektion & & $\begin{array}{l}\text { Cefuroxim } \\
\text { Amoxycillin + Clavulansäure }\end{array}$ & & Gelbe Liste Ulm 2006 \\
\hline $\begin{array}{l}\text { posttraumatische/postoperative } \\
\text { Osteitis }\end{array}$ & $\begin{array}{l}\text { Staphylokokken } \\
\text { Streptokokken } \\
\text { Enterokokken } \\
\text { Enterobakterien } \\
\text { Anaerobier } \\
\text { Mischinfektionen }\end{array}$ & $\begin{array}{l}\text { Aminopenicillin + } \beta \text {-Lak.Inhibitor } \\
\text { Cephalosporin Gr. } 2 \\
\text { Clindamycin } \\
\text { bei MRSA/MRSE: } \\
\text { Linezolid } \\
\text { Teicoplanin }\end{array}$ & $\begin{array}{l}\text { 1-4 Wochen i.v., dann } \\
\text { 2-6 Wochen p. o. } \\
\text { insgesamt mind. } 8 \text { Wochen }\end{array}$ & PEG Empfehlung 2004 \\
\hline Protheseninfekt & $\begin{array}{l}\text { Staphylokokken } \\
\text { Streptokokken } \\
\text { Enterokokken } \\
\text { Enterobakterien } \\
\text { Nonfermenter } \\
\text { Anaerobier } \\
\text { Mischinfektionen }\end{array}$ & erregerspezifische Regime & $\begin{array}{l}\text { 2-4 Wochen i.v., dann p.o. } \\
\text { insgesamt: } \\
\text { Hüftprothese: } 3 \text { Monate } \\
\text { Knieprothese: } 6 \text { Monate }\end{array}$ & Zimmerli et al. 2004 \\
\hline $\begin{array}{l}\text { fortgeleitete Osteitis nach } \\
\text { offenem Trauma oder Fraktur }\end{array}$ & & $\begin{array}{l}\text { Levofloxacin/Rifampicin } \\
\text { Cefuroxim/Rifampicin }\end{array}$ & & Gelbe Liste Ulm 2006 \\
\hline Erysipel & $\begin{array}{l}\text { Streptokokken (A, C, G) } \\
\text { selten S. aureus }\end{array}$ & $\begin{array}{l}\text { Penicillin G } \\
\text { Oxacillin } \\
\text { Cephalosporin }\end{array}$ & & \\
\hline Gasbrand & Clostridium perfringens & Penicillin $G_{ \pm}$Clindamycin & & \\
\hline
\end{tabular}

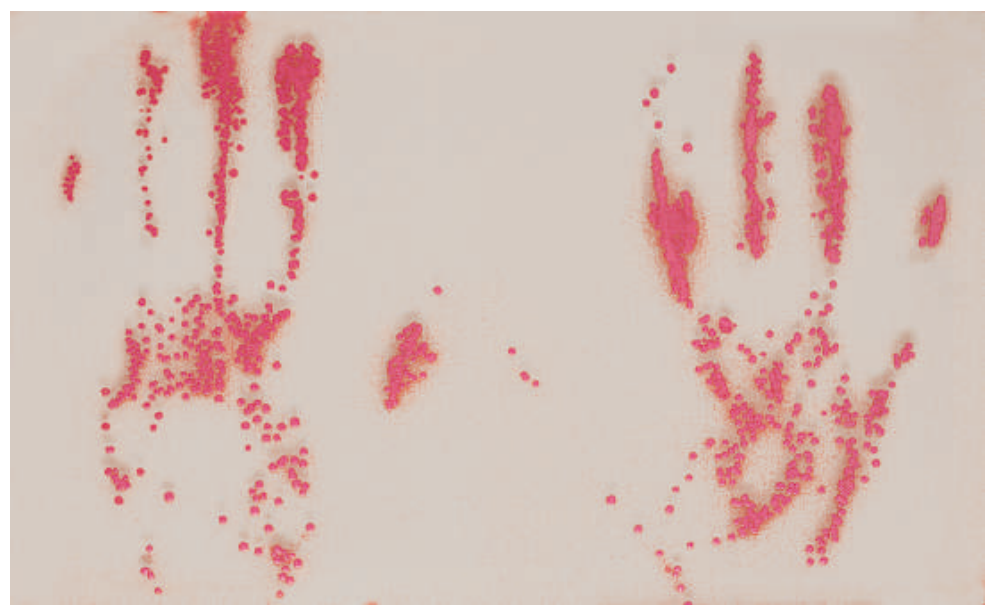

Abb. 7 - „Die kontaminierte Hand des Chirurgen“ ist nach wie vor ein wichtiger Risikofaktor für die Entstehung postoperative Wundinfektion.

- Durch Vermeiden einer postoperativen Hypothermie, perioperative Sauerstoffzufuhr, gewebeschonende, zielgerichtete Operationsverfahren, kurze Operationszeiten und den fachgerechten perioperativen Umgang mit dem Patienten kann das Risiko einer postoperativen Wundinfektion reduziert werden. Nach wie vor hat die korrekte Handhygiene oberste Priorität bei der Vermeidung noso- komialer Infektionen, da die meisten Erreger über kontaminierte Hände des medizinischen Personals übertragen werden (Abb. 7).

Bei höherem Risiko einer postoperativen Wundinfektion bei gleichzeitiger nasaler Besiedlung durch Staphylococcus aureus kann bei bestimmten Risikogruppen bei elektiven Eingriffen ein präoperatives Screening und die anschließende Eradikation diskutiert werden. Insbesondere bei Eingriffen an Gelenken ist eine strenge Indikationsstellung erforderlich und ein höchstmöglicher Hygienestandard zu fordern. Gegebenenfalls können diese Eingriffe unter Antibiotikaprophylaxe durchführt werden, dabei ist zu beachten, dass die entnommenen intraoperativen Abstriche nur eingeschränkt beurteilt werden können.

Bei offenen Frakturen spielt bereits das präklinische Management und die sachgemäße Handhabung und Antibiotikaprophylaxe in der chirurgischen Ambulanz eine wichtige Rolle.

Auch an die fachkundige und engmaschige postoperative Wundkontrolle und -pflege auf Station sind höchste Ansprüche zu stellen. Nicht zuletzt ist eine genaue Dokumentation und Infektionsstatistik wegweisend für die Optimierung der Infektprophylaxe. 


\section{Danksagung}

Wir danken Herrn PD Dr M. Bischoff (Universitätsklinikum Ulm, Zentrum für Chirurgie, Klinik für Unfallchirurgie, Hand-, Plastische und Wiederherstellungschirurgie) für die freundliche Überlassung der Vorlage für Abbildung 6.

\section{Literatur}

Chau CL, Griffith JF. Musculoskeletal infections: ultrasound appearances. Clin Radiol 2005; 60: 149-159

Costerton JW, Montanaro L, Arciola C. Bacterial communications in implant infection: a target for an intelligence war. Int J Artif Organs 2007; 30: 757-763

El-Maghraby TA, Moustafa HM, Pauwels EK. Nuclear medicine methods for evaluation of skeletal infection among other diagnostic modalities. Q J Nucl Med Mol Imaging 2006; 50: 167-192

Empfehlung der Kommission für Krankenhaushygiene und Infektionsprävention beim Robert Koch-Institut. Prävention postoperativer Infektionen im Operationsgebiet. Bundesgesundheitsbl Gesundheitsforsch Gesundheitsschutz 2007; 50: 377-393

Gelbe Liste Ulm 2006: Antiinfektiva. Leitlinien für die Therapie und Prophylaxe. Arzneimittelkommission des Universitätsklinikums Ulm (Hrsg). 9. Auflage, November 2006

Herrmann M, Becker K, von Eiff C et al. Mikrobiologisch-infektiologische Qualitätsstandards (MiQ)18: Infektionen der Knochen und des Knorpels. München: Urban und Fischer Verlag, 2004

Hofmann GO. Infektionen der Knochen und Gelenke in Orthopädie und Unfallchirurgie. 1. Aufl. München: Urban \& Fischer, 2004: 15-221f

Stumpe KD, Strobel K. 18F FDG-PET imaging in musculoskeletal infection. Q J Nucl Med Mol Imaging 2006; 50: 131 - 142

Thomas L. Labor und Diagnose. 7. Aufl. Frankfurt: Th-Books Verlagsgesellschaft, 2008
Vogel F, Bodmann KF. Expertenkommission der Paul-Ehrlich-Gesellschaft. Empfehlungen zur kalkulierten parenteralen Initialtherapie bakterieller Erkrankungen bei Erwachsenen. Chemother J 2004; 13 46- 105

Wagner C, Hänsch GM, Wentzensen A, Heppert V. Implant-associated post-traumatic osteomyelitis. Bacterial biofilms and the immune defence as protagonists of the local inflammatory process. Unfallchirurg 2006; 109: 761 - 769

Walker RC, Jones-Jackson LB, Martin W, Habibian MR, Delbeke D. New imaging tools for the diagnosis of infection. Future Microbiol 2007 2: $527-554$

Widmer AF, Francioli F. Postoperative Wundinfektionen: eine Übersicht. Swiss-Noso 1996; 3: 1 - 12

Willenegger $\mathrm{H}$, Roth $\mathrm{B}$. Treatment tactics and late results in early infection following osteosynthesis. Unfallchirurgie 1986; 12: 241 - 246 de Winter F, van de Wiele C, Vogelaers D, de Smet K, Verdonk R, Dierckx RA. Fluorine-18 fluorodeoxyglucose - position emission tomography: a highly accurate imaging modality for the diagnosis of chronic musculoskeletal infection. J Bone Joint Surg Am 2001; 83: 651 - 660 Zimmerli W, Trampuz A, Ochsner PE. Prosthetic-joint infections. N Engl J Med 2004; 351: $1645-1654$

\section{Korrespondenzadresse}

Dr. Miriam Kalbitz

Universitätsklinikum Ulm, Zentrum für Chirurgie

Klinik für Unfallchirurgie,

Hand-, Plastische und Wiederherstellungschirurgie

Steinhövelstraße 9

89075 Ulm

Telefon: $0731 / 50054570$

Telefax: 0731/50065349

E-Mail: miriam.kalbitz@uniklinik-ulm.de 


\section{CME-Fragen}

Die folgenden Fragen beziehen sich auf den vorangehenden Beitrag. Sie können uns die entsprechenden Antworten entweder online unter http://cme.thieme.de oder durch das CME-Teilnahmeheft hinten in dieser Zeitschrift zukommen lassen. Jeweils eine Antwort ist richtig.

Die Vergabe von CME-Punkten ist an die korrekte Beantwortung der Multiple-Choice-Fragen gebunden.

Welche/r Faktor/en erhöht/en das postoperative Wundinfektionsrisiko?
1. lange OP-Dauer

2. Nikotinabusus

3. Vorhandensein von offenen Frakturen und schwerem Weichteiltrauma

4. zahlreiche Revisionen

A Nur Aussage 1 ist richtig.

B Nur die Aussagen 1 und 2 sind richtig.

C Nur Aussage 3 ist richtig.

D Nur die Aussagen 3 und 4 sind richtig.

E Alle Aussagen sind richtig.
Was ist die häufigste Infektionsquelle für eine postoperative Wundinfektion?
A Luft
B Mikroorganismen im OP-Gebiet
C unbelebte Umgebung
D Personal
E bakterielle Besiedlung des Patienten außerhalb des OP-Gebietes

Die Kontamination des OP-Gebietes wird in 4 Kategorien eingeteilt. Welche Kategorie trifft nicht zu?

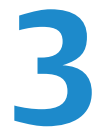
A sauber
B sauber-kontaminiert
C kontaminiert
D stark kontaminiert
E infiziert

Welche Aussage/n zu Entnahme und Transport von Material für die mikrobiologisch-infektiologische Diagnostik ist/sind richtig?
1. möglichst kontaminationsfreie Entnahme

2. oberflächliche Abstriche vermeiden, da nur geringe Aussagekraft

3. Entnahme mehrer Materialien aus einem Infektionsherd erhöht die diagnostische Sensitivität

4. unverzüglicher ( $<24 \mathrm{~h}$ ) Transport ins Labor

A Keine Aussage ist richtig.

B Nur Aussage 1 ist richtig.

C Nur Aussage 2 ist richtig.

D Nur die Aussagen 3 und 4 sind richtig.

E Alle Aussagen sind richtig.
Was ist der häufigste Erreger der postoperativen Gelenkinfektion?
A koagulasenegative Staphylokokken
B Streptokokken
C gramnegative Bakterien
D Staphylococcus aureus
E Enterokokken 


\section{Prophylaxe und Management von Komplikationen}

Was ist/sind kein/e typisches/n klinisches/n Zeichen einer postoperativen Wundinfektion?
A Rubor, Calor, Tumor, Dolor und Functio laesa

B Sekretion

C Krankheitsgefühl

D Exanthem

E Fieber
Was trifft für die Antibiotikatherapie bei postoperativen Wundinfektionen nicht zu?
A Die sofortige Verwendung eines Reserveantibiotikums ist für die Infektbeherrschung entscheidend.

B Die empirische kalkulierte Antibiotikaprophylaxe muss unter Berücksichtigung der lokalen Resistenzsituation die häufigsten Erreger der postoperativen Wundinfektion erfassen.

C Cefuroxim oder Amoxicillin plus Clavulansäure sind Mittel der ersten Wahl bei postoperativen Wundinfektionen ohne Erregernachweis.

D Bei der Therapie einer postoperativen Weichteilinfektion oder Osteomyelitis müssen Antibiotika mit guter Gewebegängigkeit und hoher Bioverfügbarkeit gewählt werden.

E Eine mikrobiologische Diagnostik ist vor Beginn der antibiotischen Therapie anzustreben.
Was trifft für das richtige Vorgehen bei drohendem Infekt nicht zu?
A Ruhigstellung
B Antibiose
C frühzeitige Entscheidung zur Revision
D Isolation des Patienten
E regelmäßige Kontrolle der laborchemischen Entzündungsparameter

Was trifft für das richtige Vorgehen bei einer postoperativen Wundinfektion nicht zu?
A Oberste Priorität hat der Erhalt der Osteosynthese.

B Die Revisionseingriffe sollten ein ausgiebiges Débridement, eine Nekrosektomie und Spülung mit 0,9 \%iger NaCl-Lösung beinhalten.

C Oberste Priorität hat die Infektbeherrschung.

D Abstriche und Gewebeproben müssen für die mikrobiologische Aufarbeitung entnommen werden.

E Nach Erhalt der Abstrichergebnisse ist die Antibiose antibiogrammgerecht anzupassen.
Was ist keine typische Komplikation einer postoperativen Wundinfektion?
A Sepsis
B Arthrose
C Pseudarthrose
D Osteomyelitis
E Kompartmentsyndrom 